\title{
PERFIL DE ASSOCIAÇÕES DE AGRICULTORES FAMILIARES DO PROGRAMA NACIONAL DO CRÉDITO FUNDIÁRIO DA ZONA DA MATA ALAGOANA ${ }^{1}$
}

\author{
Silvio Serafim de Oliveira', Elâne Rafaella Cordeiro Nunes Serafim², Maria de Fátima da Silva ${ }^{3}$, Éder Abne \\ Cordeiro Nunes ${ }^{4}$, Elvis Thiago Cordeiro Nunes ${ }^{5}$, Marcelo Cavalcante ${ }^{6}$
}

\author{
1Trabalho da dissertação do Programa de Pós-graduação em Tecnologias Ambientais (PPGTEC) - IFAL \\ ${ }^{2}$ Instituto Federal de Alagoas: Programa de Pós-graduação em Tecnologias Ambientais, Rua Lourival Alfredo, 176 - Poeira, Mal. \\ Deodoro - AL, 57160-000. E-mail: silvio.science@gmail.com; marcelo.agronomia@gmail.com \\ ${ }_{3}^{3}$ Prefeitura Municipal de Cupira, Secretaria de Saúde, Av, Etelvino Lins, s/n, Centro, Cupira - PE. E-mail:elane.rafaela@hotmail.com \\ 4Universidade Federal Rural de Pemambuco, R. Manuel de Medeiros, s/n - Dois Irmãos, Recife - PE, 52171-900. E-mail: f4tyma.adm@gmail.com \\ ${ }^{5}$ Prefeitura Municipal de Santana do Ipanema, Secretaria de Saúde, Rua, Professor Aloísio Ernandes Brandão, s/n, Camuxinga, \\ Santana do Ipanema - AL. E-mail: eder_abne@hotmail.com \\ ${ }^{6}$ Prefeitura Municipal de Maraial, Secretaria de Saúde,Rua,Laurindo do O. Santos, 26, Centro, Maraial-PE. E-mail: thiago.cordeiro19@hotmail.com
}

RESUMO: A utilização de alguns aspectos produtivos e econômicos pelos agricultores familiares pode promover uma nova visão do papel do espaço rural na luta contra as desigualdades sociais. Buscou-se identificar o perfil de associações de agricultores familiares do crédito fundiário dos municípios de União dos Palmares e Santana do Mundaú caracterizando aspectos ambientais e econômicos. Utilizou-se abordagem descritiva com natureza bibliográfica e de campo, com fins qualitativos. Realizou-se elaboração e aplicação da metodologia participativa de extensão rural para o desenvolvimento sustentável em quatro associações do crédito fundiário. Observou-se que as associações têm mais de dez anos de atividade, com em média 25 famílias, cuja propriedade é, em média, 6,0 ha, cultivados com espécies anuais e perenes, além de áreas de preservação, com a presença de nascentes e de animais (10 espécies) endêmicas da fauna da Mata Atlântica, além da criação de aves, bovinos, suínos e caprinos. Conclui-se que as associações de agricultores familiares de municípios alagoanos se assemelham quando a aspectos sociais, ambientais e econômicos.

Palavras-chave: agricultura familiar, meio ambiente, sustentabilidade

\section{PROFILE OF ASSOCIATIONS OF FAMILY FARMERS OF THE NATIONAL LAND CREDIT PROGRAM OF THE ALAGOAN FOREST ZONE}

\begin{abstract}
The use of some productive and economic aspects by family farmers can promote a new vision of the role of rural areas in the fight against social inequalities. It was sought to identify the profile of the associations of family farmers of the land credit of the municipalities of União dos Palmares and Santana do Mundaú, characterizing environmental and economic aspects. A descriptive approach with bibliographical and field nature was used for qualitative purposes. The participatory methodology of rural extension for sustainable development was elaborated and applied in four associations of land credit. It was observed that the associations have more than ten years of activity, with an average of 25 families, whose property is on average 6.0 ha, cultivated with annual and perennial species, as well as preservation areas, with the presence of springs and animals (10 species) endemic to the Atlantic Forest fauna, as well as birds, cattle, pigs and goats. It can be concluded that associations of family farmers in the municipalities of Alagoas are similar when it comes to social, environmental and economic aspects.
\end{abstract}

Keywords: family agriculture, environment, sustainability

\section{INTRODUÇÃO}

As empresas rurais são definidas como aquelas que exploram a capacidade produtiva do solo por meio do cultivo da terra, da criação de animais e da transformação de determinados produtos agrícolas (MARION, 2002). Nessas unidades, a gestão administrativa desenvolvida em seu âmbito abrange aspectos relacionados à atividade-fim, otimizando as 
atividades comerciais entre as empresas e 0 ambiente externo (CALADO; CALADO; MACHADO, 2007).

Considerando essas características, a empresa rural, familiar ou patronal necessita adaptarse às exigências de mercado e produção (BATALHA et al., 2004), adotando sistemas de mensuração e indicadores de desempenho que possibilitem o planejamento e a implantação de estratégias pelo seu corpo, de modo a auxiliar a gestão e a manutenção dos seus negócios, ampliando a visão de mercado do produtor (CALADO;CALADO; MACHADO, 2007).

O Programa Nacional de Crédito Fundiário (PNCF) oferece condições para que os trabalhadores e trabalhadoras sem terra ou com pouca terra possam comprar imóvel rural por meio de um financiamento. Além da terra, os recursos financiados podem ser utilizados na estruturação da propriedade e do projeto produtivo, na contratação Assistência Técnica e Extensão Rural (Ater) e no que mais for necessário para que 0 agricultor venha desenvolver suas atividades de forma independente e autônoma (MDA, 2018).

Portanto, ao considerar que as estratégias utilizadas nas organizações locais para agricultura familiar, envolvendo aspecto produtivo e de desenvolvimento descentralizado, podem ser os embriões que irão promover uma nova visão do papel do espaço rural na luta contra as desigualdades sociais em outras regiões (ABRAMOVAY, 1997), buscouse identificar o perfil de associações de agricultores familiares do Programa Nacional do crédito fundiário em União dos Palmares e Santana do Mundaú, caracterizando aspectos ambientais e econômicos.

\section{MATERIAL E MÉTODOS}

A área de estudo está inserida na mesorregião do Leste Alagoano, na Zona da Mata, caracterizandose por apresentar relevo ondulado e por ser percorrida pelo alto curso do rio Mundaú (IBGE, 1997). Nessa região a agricultura familiar representa mais de $70 \%$ das propriedades em quase todos os municípios. Em União dos Palmares representa 92,3\% e em Santana do Mundaú, 48,2\% dos estabelecimentos (BRASIL, 2011).

Esta pesquisa foi de abordagem descritiva com fins qualitativo, realizada no período de setembro a dezembro de 2015. O estudo foi realizado em quatro associações de agricultores familiares beneficiados pelo Programa Nacional do Crédito Fundiário (PNCF), $A$ e B, localizadas no município de Santana do Mundaú, C e D, em União dos Palmares.

Para elaboração e aplicação do questionário, envolvendo características sócioambientais, utilizou-se a metodologia participativa de extensão rural para 0 desenvolvimento sustentável (MEXPAR) (RUAS et al., 2006).

Os dados foram coletados em dois momentos distintos. 0 primeiro foi realizado em atividade coletiva com a comunidade, utilizando como instrumento o diagnóstico rural coletivo. 0 outro momento ocorreu a partir de visitas individuais nos lotes dos associados, utilizando como instrumento o diagnóstico rural individual, totalizando 99 visitas.

\section{RESULTADOS E DISCUSSÃO}

As associações de agricultores familiares beneficiadas possuem mais de dez anos de atividade, apresentando aproximadamente 6 ha de área de terra cada, como demonstrado na Tabela 1.

Tabela 1. Caracterização social das associações de agricultores nos municípios alagoanos, Santana do Mundaú e União dos Palmares.

\begin{tabular}{lccccc}
\hline & \multirow{2}{*}{ Variáveis } & \multicolumn{2}{c}{ SM } & \multicolumn{3}{c}{ UP } \\
\cline { 2 - 5 } & $\mathrm{A}$ & $\mathrm{B}$ & $\mathrm{C}$ & $\mathrm{D}$ \\
\hline Anos de atividade & 14 & 14 & 11 & 13 \\
No de Famílias & 30 & 30 & 20 & 19 \\
Área total (ha) & 200 & 200 & 115 & 154 \\
Área média por família (ha) & 6 & 5,7 & 5,7 & 6 \\
Habitação & Sim & Sim & Sim & Sim \\
Saneamento & Não & Não & Não & Não \\
Saúde & Sim & Sim & Sim & Sim \\
Educação & Sim & Sim & Não & Não \\
\hline
\end{tabular}

SM: Santana do Mundaú; UP: União dos Palmares; 
Observou-se que, em média, 25 famílias residem nas associações de agricultores familiares, e não há variação da quantidade de unidades familiares nas associações por município.

Em relação à oferta de educação na própria área da associação, há escolas instaladas e ativas em Santana do Mundaú para séries iniciais, e em União dos Palmares os alunos são deslocados, através de transporte municipal, para escolas localizadas em outras áreas. Em todas as localidades as residências são de alvenaria e não tem saneamento básico. Esses aspectos são considerados fatores determinantes e condicionantes da saúde, pois se enquadram em condições gerais de ordem socioeconômica, cultural e ambiental de uma sociedade e se relacionam com as condições de vida e trabalho de seus membros (BRASIL, 2009).

As questões de moradia e saúde também estão relacionadas com a satisfação dos associados com as necessidades básicas individuas, favorecendo sua permanência no campo, pois é considerada uma das alternativas do problema da produção de alimentos no mundo, uma vez que a agricultura familiar em formato associativista é capaz de atender uma parcela significativa da necessidade da população (TONIASSO et al., 2007).

Em relação às características ambientais identificadas nas associações, observaram-se áreas de vegetação que se caracterizam como pertencente à Mata Atlântica, e a presença de área de preservação em todas as comunidades, como demonstrado na Tabela 2.

Tabela 2. Caracterização Ambiental das associações de agricultores nos municípios alagoanos, Santana do Mundaú e União dos Palmares.

\begin{tabular}{lcccc}
\hline & \multirow{2}{*}{ Itens } & \multicolumn{2}{c}{ SM } & UP \\
\cline { 2 - 5 } & A & B & C & D \\
\hline Vegetação & Atla & Atla & Atla & Atla \\
Área preservação (ha) & 40 & 40 & 29 & 30 \\
Barreiro & 1 & 1 & 3 & - \\
Barragem & 1 & 0 & - & - \\
Rio & - & - & - & 1 \\
Nascente & 15 & 12 & 8 & 12 \\
\hline
\end{tabular}

SM: Santana do Mundaú; UP: União dos Palmares; Atla: mata Atlântica

0 cuidado com essas áreas de preservação garante as condições gerais de estabilidade entre as atividades produtivas e o meio ambiente, favorecendo a sobrevivência das populações locais (TONIASSO et al., 2007), principalmente pela trajetória histórica de exploração do território em estudo, que ocasionou uma redução 93,96\% da extensão original dessa vegetação
(MOURA, 2006).

Em relação aos animais no país há registro de 524 espécies de mamíferos, 69 destas já foram relatadas na Mata Atlântica que abrange a área de estudo, o Estado Alagoas (MOURA, 2006). E nas áreas estudadas, 10 espécies de mamíferos foram mencionadas, conforme exposto na Tabela 3.

Tabela 3. Levantamento de mamíferos relatados nas associações de agricultores nos municípios alagoanos, Santana do Mundaú e União dos Palmares.

\begin{tabular}{|c|c|c|c|c|}
\hline \multirow{2}{*}{ Espécie de animais } & \multicolumn{2}{|c|}{ SM } & \multicolumn{2}{|c|}{ UP } \\
\hline & $A$ & $B$ & $\mathrm{C}$ & $D$ \\
\hline Bradypus variegatus $^{1}$ & + & - & + & + \\
\hline Callithrix jacchus ${ }^{2}$ & + & - & + & + \\
\hline Cavia aperea ${ }^{3}$ & - & + & - & - \\
\hline Chrysocyon brachyurus ${ }^{4}$ & - & + & - & - \\
\hline Cuniculus paca ${ }^{5}$ & - & + & - & - \\
\hline Dusicyon thous ${ }^{6}$ & + & + & - & + \\
\hline Euphractus sexcinctus ${ }^{7}$ & - & + & + & + \\
\hline Leopardus tigrinus $^{8}$ & - & + & - & - \\
\hline Lontra longicaudis $^{9}$ & - & + & - & - \\
\hline Sylvilagus brasiliensis ${ }^{10}$ & - & + & - & - \\
\hline
\end{tabular}

SM: Santana do Mundaú; UP: União dos Palmares; (+): existente; (-): ausente; 1: Preguiça-comum; 2: Sagui-de-tufobranco; 3: Preá; 4:Lobo-Guara; 5: Paca; 6: cachorro-do-mato; 7: Tatupeba; 8: Gato-do-mato; 9 : Lontra; 10: Lebre. 
A distribuição desses animais foi semelhante entre as associações e os municípios pesquisados. A maioria das espécies animais ou vegetais que vivem nos fragmentos de Mata Atlântica compõe populações isoladas de populações que habitam outros fragmentos (MOURA, 2006).

0 desmatamento generalizado também pode ocasionar redução da produção de água nas nascentes (BAGGIO et al., 2013). Observou-se que nas associações avaliadas os recursos hídricos predominante são as nascentes. Assim, recomenda-se, proteger as nascentes para garantir a água adequada para consumo humano, e evitar o acesso direto de animais de criação às nascentes, córregos e barragens instalando bebedouros com bóias do lado de fora da cerca que delimita a aguada (BAGGIO et al., 2013).

A economia das associações é baseada principalmente no plantio e comercialização de raízes, frutas, hortaliças e feijão, e em menor quantidade, na criação e comercialização de animais como aves, bovinos, suínos e caprinos. A dinâmica dessas atividades precisa incorporar determinações geradas pela globalização com a conservação dos recursos materiais e com a autosustentabilidade da produção agrícola, situação que também pode favorecer 0 crescimento da renda desse segmento exercendo papel fundamental para o desenvolvimento social, evitando o êxodo rural e tornando-se instrumento de inclusão social, geração de trabalho e renda (GOBBl; PESSÔA, 2009).

Vale ressaltar que a região avaliada pauta seus valores e princípios no protagonismo do fortalecimento da agricultura familiar por meio do associativismo e o cooperativismo, com a implementação de políticas públicas voltadas para o desenvolvimento do meio rural, buscando o resgate da cultura local, a ampliação e diversificação da produção agropecuária em bases agroecológicas e a preservação do meio ambiente (BRASIL, 2011).

\section{CONCLUSÕES}

As associações de agricultores familiares de municípios alagoanos se assemelham quando a aspectos sociais, ambientais e econômicos. 0 perfil dessas associações serve como suporte na elaboração de planos e estratégias que vise o desenvolvimento da produção familiar em comunidades rurais a partir da relação de aspectos ambientais e produtivos de uma localidade.

\section{AGRADECIMENTOS}

Associados do Programa Nacional do Crédito Fundiário de Santana do Ipanema e União dos Palmares.

\section{REFERÊNCIAS}

ABRAMOVAY, R. Agricultura familiar e uso do solo. São Paulo em Perspectiva, 1997. p. 73-78,

BAGGIO, A.J.; CARPANEZII, A.A.; FELIZARI, S.R., RUFFATO, A. Recuperação e proteção de nascente em propriedades rurais de Machadinho, RS. Brasília: Embrapa, 2013, 26p.

BATALHA, M.O.; SOUZA FILHO, H.M.; BUAINAIM, A.M. Tecnologia de gestão e agricultura familiar. In: XLII Congresso Brasileiro de Economia e Sociologia Rural - SOBER, 2004, Cuiabá. Dinâmicas setoriais e desenvolvimento regional. Rio de Janeiro: SOBER, 2004.

BRASIL. Plano Territorial de Desenvolvimento Rural Sustentável do Território da Cidadania Mata Alagoana. Brasília: Ministério do Desenvolvimento Agrário. 2011. $96 \mathrm{p}$.

BRASIL. Ministério da Saúde. Saúde na escola/ Ministério da Saúde, Secretaria de Atenção à Saúde, Departamento de Atenção Básica. - Brasília: Ministério da Saúde, 2009. 96p.

CALLADO, A.A.C; CALLADO, A.L.C. MACHADO, M.A.V. Indicadores de desempenho operacional e econômico: um estudo exploratório no contexto do agronegócio. Revista de Negócios, 2007, 12, 3-15.

GOBBI, W.A.O.; PESSÔA; V.L.S. A pecuária leiteira e a agricultura familiar em Ituiutaba (MG): as transformações na comunidade da canoa. GEO UERJ, $2009,2,79-110$.

GUILHOTO, J.J.M.; SILVEIRA, F.G.; ICHIHARA, S.M.; AZZONI, C.R. A importância do agronegócio familiar no Brasil. Revista de Economia e Sociologia Rural, 2006, 44, 355-382. 
MARION, J.C. Contabilidade rural. 7ed. São Paulo: RUAS, E.D.; BELTRÃO, I.M. de M.; CARVALHO, Atlas, 2002. 165p. M.A.T.; SOARES, M.H.P. Metodologia Participativa da Extensão Rural para o Desenvolvimento Sustentável -

MDA: Ministério do Desenvolvimento Agrário. Programa MEXPAR. $1^{\text {a }}$ ed. Belo Horizonte: Bárbara Bela Editora Nacional de Crédito Fundiário. Disponível em: <http:/l www.mda.gov.br/sitemda/secretaria/sra-crefun>. Acesso em: 05 Nov. 2018. Gráfica, 2006.

TONIASSO, H.R.; SOUZA, C.C.; BRUM, E; FIGUEIREDO, R.S. Agricultura Familiar e MOURA, F. B. P. org. A Mata Atlântica em Alagoas. Associativismo Rural - o caso associação harmonia Maceió: EDUFAL, 2006. 88p. de agricultura familiar de Mato Grosso do Sul e a sua sustentabilidade. Informe GEPEC, 2007. 2.

ZYLBERSZTAJN, D. Organização de cooperativas: desafios e tendências. Revista de Administração, 1994, 29, 23-32. 